\title{
A NEW EXPRESSION FOR RADIAL DISTRIBUTION FUNCTION OF NUCLEAR MATTER
}

\author{
Fatma Manisa $^{1}$, Atalay Küçükbursa ${ }^{1 *}$, Kaan Manisa ${ }^{1}$ and Tahsin Babacan ${ }^{2}$ \\ ${ }^{1}$ Dumlupınar University, Department of Physics, Kütahya, Turkey \\ ${ }^{2}$ Celal Bayar University, Department of Physics, Manisa, Turkey \\ atalay@dumlupinar.edu.tr
}

\begin{abstract}
A Variational Monte Carlo Method (VMC) is used for the calculations of radial distribution function of nuclear matter. Urbana $v_{14}$ potential is used for the nucleon-nucleon interactions in the calculations. The new expression for radial distribution function of nuclear matter is obtained by fitting of Monte Carlo simulations results to a function.
\end{abstract}

Key Words-Nuclear matter, radial distribution function, Monte Carlo method.

\section{INTRODUCTION}

The radial distribution function (RDF) is very important in the theory of N-body systems because it gives the number of the particle between $r$ and $r+d r$ about a central particle at the origin of $\mathrm{r}$ and it can also be thought of as the factor that multiplies the bulk density, $\rho$, to give a local density $\rho(r)=\rho g(r)$ about some fixed particle. Another important point is that RDF provides a sufficient information in calculating all the thermodynamic functions of the system.

The most reliable calculations of the radial distribution function are molecular dynamics or Monte Carlo calculations. One advantage of these calculation methods is that the inter-particle potential is known. Therefore, these calculations serve as "experimental" data which is used to test various theories [1]. For hard spheres, the molecular dynamics radial distribution function was first given by Alder and Hecht [2], and Barker, Watts and Henderson [3]. It has been calculated for the Lennard-Jones potential by Verlet [4], and for an inverse 12 potential by Hansen and Weis [5]. Moreover, Barker, Fisher and Watts [6] have presented extensive Molecular Dynamic and Monte Carlo calculations with three body forces. They are useful in the perturbation and transport theories [7]. Recently, molecular dynamic simulations have used the radial distribution function for Lennard-Jones fluids [8, 9], and mixing rules for binary Lennard-Jones chains have been tested by performing Monte Carlo simulations [10]. The radial distribution function can also be determined by X-ray diffraction studies [1113]. However, various methods and techniques have been used to obtain the radial distribution function [14-16]. In this paper, the radial distribution function for nuclear matter is calculated by using the variational Monte Carlo method (VMC) with various isospin asymmetry parameters, $\beta$ at different densities. Moreover, the new expression for radial distribution function of nuclear matter is also obtained by using Monte Carlo simulations results.

This paper is organized as follows: the interaction potential and symmetric, asymmetric nuclear matter is define and is determined in Section 2 and in Section 3, 
respectively. In section 4, Monte Carlo simulations are described. The results for the application of the Variational Monte Carlo method to the calculation of the radial distribution function of nuclear matter are given in section 5 . Then we conclude in section 6 .

\section{INTERACTION POTENTIAL}

The Hamiltonian operator of a system consisting of $\mathrm{N}$ particles can be written as

$$
\mathbf{H}=-\frac{\hbar^{2}}{2 m} \sum_{i} \nabla_{i}^{2}+\sum_{i<j} V_{i j}
$$

where $\mathrm{V}_{\mathrm{ij}}$ is a two body interaction potential. Here, we have used only the first four terms of the Urbana $\mathrm{V}_{14}$ potential [17] for two nucleon interaction in our calculations. Because the contributions of latter terms of the Urbana $V_{14}$ potential are much smaller than those of the first four terms in our nuclear matter calculations, 14 operator components of the Urbana $\mathrm{V}_{14}$ potential are necessary to have a good fit to the experimental data. Thus, we have used the two-nucleon interaction

$$
V_{i j}=V^{c}+V^{\sigma}\left(\sigma_{i} \cdot \sigma_{j}\right)+V^{\tau}\left(\tau_{i} \cdot \tau_{j}\right)+V^{\sigma \tau}\left(\sigma_{i} \cdot \sigma_{j}\right)\left(\tau_{i} \cdot \tau_{j}\right)
$$

where $V^{c}, V^{\sigma}, V^{\tau}$, and $V^{\sigma \tau}$ depend only on the distance between the nucleons $i$ and $j$. Each term in Eq. (2) has three parts

$$
V^{i}=V_{\pi}^{i}+V_{I}^{i}+V_{S}^{i}
$$

representing the long-range interactions $\left(V_{\pi}^{i}\right)$, the intermediate-range interactions $\left(V_{I}^{i}\right)$, and the short-range $\left(V_{S}^{i}\right)$ interactions. $\left(V_{\pi}^{i}\right)$ is nonzero only for $i=\sigma \tau$ and is given by

$$
V_{\pi}^{\sigma \tau}=3.488 \frac{e^{-\mu r}}{\mu r}\left(1-e^{-c r^{2}}\right)
$$

where $\mu=0.7 \mathrm{fm}^{-1}$ is the inverse compton wavelength for pions. The intermediate and short range parts are

$$
V_{I}^{i}(r)=I^{i}\left[\left(1+\frac{3}{\mu r}+\frac{3}{(\mu r)^{2}}\right) \frac{e^{-\mu r}}{\mu r}\left(1-e^{-c r^{2}}\right)\right]^{2},
$$

and

$$
V_{S}^{i}(r)=\frac{S^{i}}{1+e^{(r-R) / a}},
$$

respectively. The values of the potential strengths $\mathrm{I}^{\mathrm{i}}$ and $\mathrm{S}^{\mathrm{i}}$ and the parameters $\mathrm{c}, \mathrm{R}$, a are given in Table I.

Table 1:. Parameters of the Urbana $V_{14}$ nucleon-nucleon potential.

\begin{tabular}{lll}
\hline$I$ & $I^{i}$ & $S^{i}$ \\
\hline $\mathrm{c}$ & -5.7030 & 2575.3 \\
$\sigma$ & 0.7628 & -366.56 \\
$\tau$ & 0.8892 & -466.56 \\
$\sigma \tau$ & -0.2790 & 402.81 \\
\hline \multicolumn{2}{c}{$c=0.2 \mathrm{fm}^{-2}, R=0.5 \mathrm{fm}, a=0.2 \mathrm{fm}$} &
\end{tabular}


Another important point is that three and more body interactions are very important in nuclear matter calculations. Therefore, we use the phenomenological approach assuming the density dependent term to be proportional to the short ranged part of the Urbana potential, and assume that the total interaction, including the many body effects, is of the form

$$
V_{14}+T N I=v_{\pi}+v_{I}+v_{s}+v_{s}(\alpha \rho)^{\gamma}
$$

where $\rho$ is the number density of nucleons. $\alpha$ and $\gamma$ in the above equation are free parameters.

\section{SYMMETRIC AND ASYMMETRIC NUCLEAR MATTER}

Nuclear matter is an idealized system of interacting nucleons (protons and neutrons). It is not matter in a nucleus, but a hypothetical system consisting of a huge number of protons and neutrons interacting by only nuclear force and no Coulomb force. Volume and particle numbers are infinite, but the ratio of these quantities is finite. Infinite volume implies no surface effects and translational invariance (only differences in position matter, not absolute positions).

A common idealization is a symmetric nuclear matter which consists of equal numbers of protons and neutrons. The energy per particle of the asymmetric nuclear matter can be expanded about symmetric nuclear matter in a Taylor series in terms of the isospin asymmetry parameter $\beta$

$$
E(\rho, \beta)=E(\rho)+\beta^{2} S_{1}(\rho)+\beta^{4} S_{2}(\rho)+\ldots,
$$

If the fourth order and higher order terms in $\beta$ are ignored, then the total energy per particle of asymmetric nuclear matter can be written as:

$$
E(\rho, \beta)=E_{s}(\rho)+\beta^{2} S(\rho),
$$

where $E_{s}(\rho)$ is the energy per nucleon of symmetric nuclear matter, $\beta=\frac{N_{n}-N_{p}}{N_{n}+N_{p}}$ is the isospin asymmetry parameter (where $N_{n}$ and $N_{p}$ are the numbers of neutrons and protons) and $S(\rho)$ is called as the symmetry energy. A detailed explanation and information for the properties of the symmetric and asymmetric nuclear matter can be found in our previous publications $[18,19]$.

\section{MONTE CARLO SIMULATIONS}

We will consider a system of nucleons confined in a cube of side $\mathrm{L}$ with periodic boundaries. The size of the cube determined from the density and number of particles was chosen so as to correspond to a closed shell in k-space representing all the symmetries of the ground state. One must use the fully occupied closed shells of plane waves for both neutrons and protons in order to preserve the isotropy of the system. The number of spin-isospin degeneracy of the spatial states is denoted by $\mathrm{g}$ ( $\mathrm{g}=2$ for proton and neutron matter). Number of spatial states in each shell is given in Table 1. For our wave function, in order to represent all symmetries of the ground state, the number of spatial states (I) should be chosen from the series of $\mathrm{I}=1,7,19,27,33,57,81, \ldots$, so that 
a complete shell in momentum space must be filled. Thus, the number of neutrons or protons must be chosen from the set of $2,14,38,54,66,114, \ldots$. Then the total number of nucleons in nuclear matter becomes $\mathrm{N}=\mathrm{g}\left(\mathrm{I}_{\mathrm{n}}+\mathrm{I}_{\mathrm{p}}\right)$. The chosen $\mathrm{I}_{\mathrm{n}}, \mathrm{I}_{\mathrm{p}}$ and the total number of nucleons are given in Table 2.

Table 2: Number of the spatial states in each shell. The number of nucleons were chosen in accordance with this table.

\begin{tabular}{|c|c|c|c|c|c|c|c|c|}
\hline$\left.n\right|^{2}$ & & $\overline{0}$ & 1 & 2 & 3 & 4 & 5 & 6 \\
\hline \multirow{24}{*}{$\begin{array}{l}\text { Allowed } \\
\text { values }\end{array}$} & \multirow[t]{24}{*}{$n_{x}, n_{y}, n_{z}$} & \multirow[t]{24}{*}{$\overline{0,0,0}$} & $1,0,0$ & $1,1,0$ & $1,1,1$ & $2,0,0$ & $0,1,2$ & $1,1,2$ \\
\hline & & & $0,1,0$ & $1,0,1$ & $-1,1,1$ & $0,2,0$ & $1,0,2$ & $1,2,1$ \\
\hline & & & $0,0,1$ & $0,1,1$ & $1,-1,1$ & $0,0,2$ & $2,1,0$ & $2,1,1$ \\
\hline & & & $-1,0,0$ & $1,-1,0$ & $1,1,-1$ & $-2,0,0$ & $0,2,1$ & $-1,1,2$ \\
\hline & & & $0,-1,0$ & $1,0,-1$ & $-1,-1,1$ & $0,-2,0$ & $1,2,0$ & $1,-1,2$ \\
\hline & & & $0,0,-1$ & $0,1,-1$ & $-1,1,-1$ & $0,0,-2$ & $2,0,1$ & $1,1,-2$ \\
\hline & & & & $-1,1,0$ & $1,-1,-1$ & & $0,-1,2$ & $-1,2,1$ \\
\hline & & & & $-1,0,1$ & $-1,-1,-1$ & & $-1,0,2$ & $1,-2,1$ \\
\hline & & & & $0,-1,1$ & & & $2,-1,0$ & $1,2,-1$ \\
\hline & & & & $-1,-1,0$ & & & $0,2,-1$ & $-2,1,1$ \\
\hline & & & & $-1,0,-1$ & & & $-1,2,0$ & $2,-1,1$ \\
\hline & & & & $0,-1,-1$ & & & $2,0,-1$ & $2,1,-1$ \\
\hline & & & & & & & $0,1,-2$ & $-1,-1,2$ \\
\hline & & & & & & & $1,0,-2$ & $-1,1,-2$ \\
\hline & & & & & & & $-2,1,0$ & $1,-1,-2$ \\
\hline & & & & & & & $0,-2,1$ & $-1,-2,1$ \\
\hline & & & & & & & $1,-2,0$ & $-1,2,-1$ \\
\hline & & & & & & & $-2,0,1$ & $1,-2,-1$ \\
\hline & & & & & & & $0,-1,-2$ & $-2,-1,1$ \\
\hline & & & & & & & $-1,0,-2$ & $-2,1,-1$ \\
\hline & & & & & & & $-2,-1,0$ & $2,-1,-1$ \\
\hline & & & & & & & $0,-2,-1$ & $-1,-1,-2$ \\
\hline & & & & & & & $-1,-2,0$ & $-1,-2,-1$ \\
\hline & & & & & & & $-2,0,-1$ & $-2,-1,-1$ \\
\hline States in & Shell & 1 & 6 & 12 & 8 & 6 & 24 & 24 \\
\hline States up & iis Shell & 1 & 7 & 19 & 27 & 33 & 57 & 81 \\
\hline
\end{tabular}

Table 3: The numerical values used in Monte Carlo simulations

\begin{tabular}{lllccccc}
\hline \multicolumn{1}{c}{$\beta$} & $\mathrm{n}_{\mathrm{p}}$ & $\mathrm{I}_{\mathrm{p}}$ & Number of proton & $\mathrm{n}_{\mathrm{n}}$ & $\mathrm{I}_{\mathrm{n}}$ & Number of neutron & Number of total nucleon \\
\hline 0.9 & 1 & 1 & 2 & 3 & 19 & 38 & 40 \\
0.78124 & 2 & 7 & 14 & 6 & 57 & 114 & 128 \\
0.75 & 1 & 1 & 2 & 2 & 7 & 14 & 16 \\
0.58824 & 2 & 7 & 14 & 4 & 27 & 54 & 68 \\
0.5 & 3 & 19 & 38 & 6 & 57 & 114 & 152 \\
0.35714 & 4 & 27 & 54 & 6 & 57 & 114 & 104 \\
0.26924 & 3 & 19 & 38 & 5 & 33 & 66 & 92 \\
0.17392 & 3 & 19 & 38 & 4 & 27 & 54 & 120 \\
0.1 & 4 & 27 & 54 & 5 & 33 & 66 & 132 \\
0 & 5 & 33 & 66 & 5 & 33 & 66 & \\
\hline
\end{tabular}


Monte Carlo simulations for nuclear matter with various isospin asymmetry parameters were carried out using the Metropolis algorithm [20] and a cubic box of side $\mathrm{L}$ containing $\mathrm{N}$ nucleons with periodic boundary conditions. The trial wave function used is a Jastrow type wave function in the form

$$
\Psi_{j}(\vec{R})=\prod_{i<j} f_{j}\left(r_{i j}\right) \Phi,
$$

where $\Phi$ is the many particle wave function for the system of non-interacting particles and $\vec{R}$ is a $3 \mathrm{~N}$ dimensional vector representing the coordinates of particles, while $f_{j}$ is the two particle correlation function. Jastrow suggests that in general, this correlation function can be an operator function [21]. Howeve, in most applications $f_{j}$ is assumed to depend only on the interparticle distance, $r_{i j}=\left|r_{i}-r_{j}\right|$.

One can use plane waves $\phi(\vec{r})=e^{i \vec{k} \cdot \vec{r}}$ for the single particle wave functions of the nucleons in bulk matter. Because we consider nucleons restricted to a cubic box of side $\mathrm{L}, \vec{k}=2 \pi \vec{n} / L$ and $\vec{n}$ is an integer vector. In order to conserve the rotational invariance of bulk nuclear matter, the number of neutrons and protons in the box is restricted to completely filled shells only. Under these conditions, the many particle wave function in eq.(9) becomes

$$
\Phi(R)=D^{P \uparrow} D^{P \downarrow} D^{N \uparrow} D^{N \downarrow}
$$

where $D^{P \uparrow}, D^{P \downarrow}, D^{N \uparrow}$ and $D^{N \downarrow}$ are the slater determinants of single particle wave functions for the corresponding spin, isospin state with

$$
D^{s}=\operatorname{det}\left(d_{i j}^{s}\right) \text {, }
$$

where

$$
d_{i j}^{s}=\phi_{j}\left((\vec{r}, s)_{i}\right) .
$$

The nuclear forces are short ranged and saturate very quickly, thus the radial distribution function is not expected to have long range correlations. Therefore, for the two particle correlation function $f_{\mathrm{j}}$ in eq.(9), a function in the following form is used

$$
f_{j}(r)=\left[\frac{1}{1+e^{\left(r_{0}-r\right) / a}}\right]^{t},
$$

where $\mathrm{t}, \mathrm{r}_{0}$ and a are the variational parameters. A pseudo potential $u(r)$ for practical reasons is defined such that our variational wave function $\left(f_{j}\left(r_{i j}\right)=\exp \left(-u\left(r_{i j}\right)\right)\right)$ becomes

$$
\Psi_{j}=\exp \left(-\sum_{i\langle j} u\left(r_{i j}\right)\right) D^{P \uparrow} D^{P \downarrow} D^{N \uparrow} D^{N \downarrow} .
$$

the $3 \mathrm{~N}$ dimensional space with the probability distribution

$$
\frac{|\Psi(R)|^{2}}{\int d R|\Psi(R)|^{2}}
$$

Is sampled by using a random walk created by the usual Metropolis method. Ceperley et.al.[22] have described the most effective way to handle the ratio of the determinants in this wave function. 
The VMC simulation methodology has been reported before in $[18,19,22]$ and the details can be found in those references.

\section{RESULTS}

In this section, the results obtained from Monte Carlo simulations for nuclear matter with various isospin asymmetry parameters are presented. The radial distribution functions of nuclear matter at densities between $0.02 \mathrm{fm}^{-3}$ and $0.20 \mathrm{fm}^{-3}$ in 0.02 steps for each isospin asymmetry parameter and a new expression for radial distribution function of nuclear matter from these data have been obtained.

The new expression for RDF of nuclear matter is found by fitting the results obtained from VMC simulations to a function:

$$
g(r)=a[1-\exp (-b r)]^{c} \text {. }
$$

In Fig. 1, we plot the selected radial distribution functions of nuclear matter with various isospin asymmetry parameters at densities between $0.02 \mathrm{fm}^{-3}$ and $0.20 \mathrm{fm}^{-3}$, calculated with VMC method. All parameters a, b and c at each density in Eq. (16) used in fitting. are given in Table 4.

It can be seen from Fig. 1 that radial distribution functions rapidly approach zero at shorter distance than $0.3 \mathrm{fm}$. This effect caused from short range repulsive parts of nucleon-nucleon interactions. However, all of radial distribution functions approach to asymptotic value of $1 \mathrm{fm}$ at shorter than $5 \mathrm{fm}$ value. Radial distribution functions reach to this asymptotic value in short range so long as the density increases. Also, radial distribution functions almost display the same behavior between 0 and $1 \mathrm{fm}$ range. Fluctuation that is between 1.5 and $3 \mathrm{fm}$ range in radial distribution functions takes smooth forms as so to be approached to symmetric nuclear matter $(\beta=0)$. 


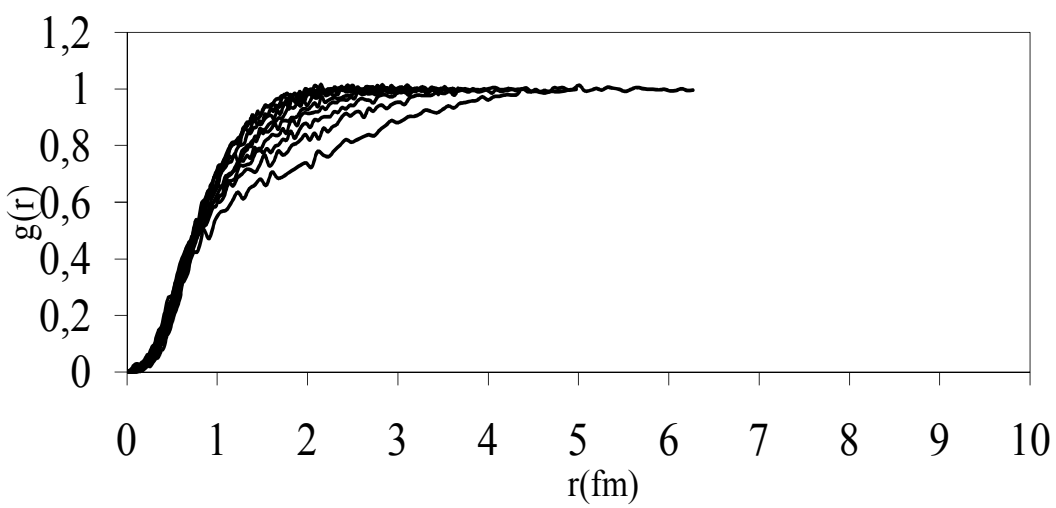

(a)

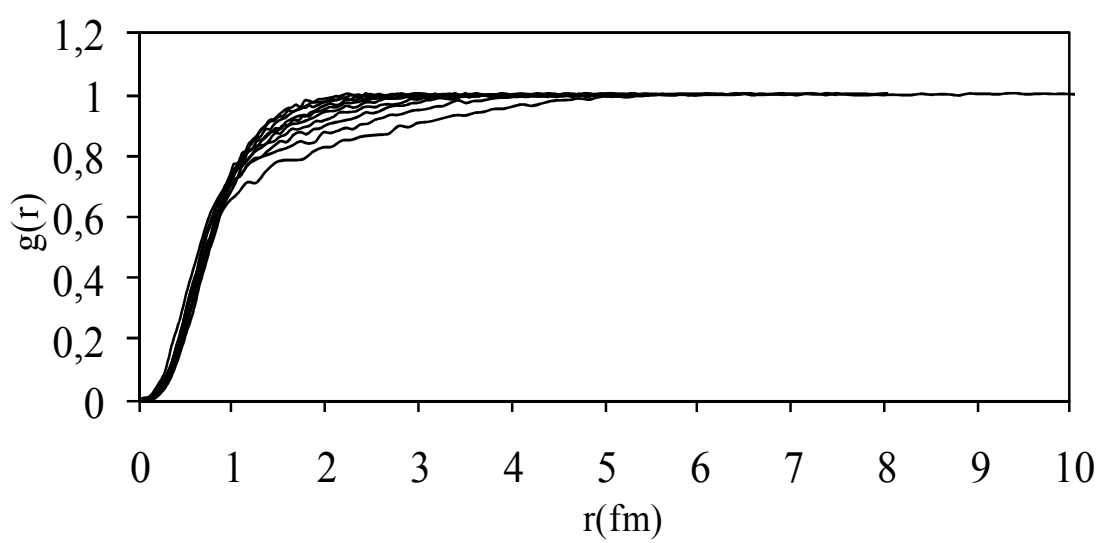

(b)

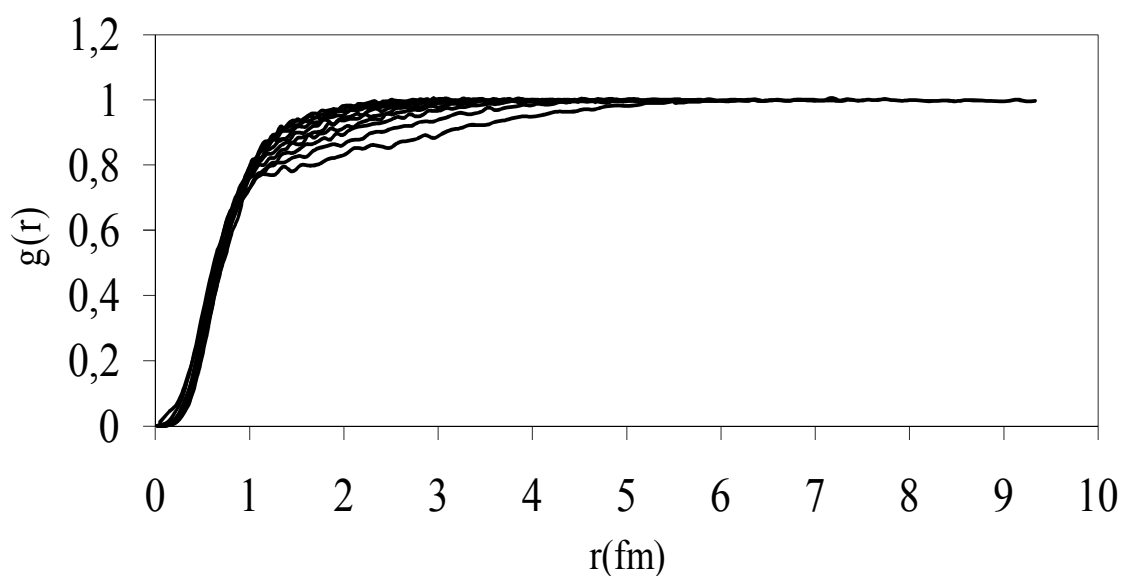

(c)

Figure 1. Nuclear matter radial distribution functions for $\beta=0.9$ (a), $\beta=0.35714$ (b) and $\beta=0$ (c). The density values increase from bottom to top with the lowermost curve corresponding to $0.02 \mathrm{fm}^{-3}$ and the uppermost one corresponding to $0.20 \mathrm{fm}^{-3}$. 
Table 4. Coefficients of the expression for RDF of nuclear matter

\begin{tabular}{|c|c|c|c|}
\hline \multicolumn{4}{|c|}{$\mathrm{YP}=0.5$ Symmetric nuclear matter } \\
\hline$\rho\left(f m^{-3}\right)$ & $\mathrm{a}$ & $\mathrm{b}$ & $\mathrm{c}$ \\
\hline 0.02 & 0,9818 & 1,1872 & 1,2279 \\
\hline 0.04 & 0,9810 & 1,7235 & 1,8207 \\
\hline 0.06 & 0,9834 & 2,2151 & 3,2190 \\
\hline 0.08 & 0,9832 & 2,4556 & 3,2489 \\
\hline 0.10 & 0,9854 & 2,6501 & 3,7443 \\
\hline 0.12 & 0,9868 & 2,9113 & 4,6280 \\
\hline 0.14 & 0,9915 & 2,8519 & 4,6306 \\
\hline 0.16 & 0,9914 & 3,2981 & 6,7760 \\
\hline 0.18 & 0,9922 & 3,3361 & 6,5198 \\
\hline 0.20 & 0,9945 & 3,3575 & 6,6010 \\
\hline \multicolumn{4}{|c|}{$\mathrm{YP}=0.45$} \\
\hline 0.02 & 0,9791 & 1,2890 & 1,4885 \\
\hline 0.04 & 0,9805 & 1,6631 & 1,7384 \\
\hline 0.06 & 0,9818 & 1,9883 & 2,1476 \\
\hline 0.08 & 0,9837 & 2,3224 & 2,9997 \\
\hline 0.10 & 0,9883 & 2,2476 & 2,6306 \\
\hline 0.12 & 0,9947 & 2,3457 & 3,4888 \\
\hline 0.14 & 0,9907 & 2,9210 & 5,1770 \\
\hline 0.16 & 0,9959 & 2,7497 & 4,5878 \\
\hline 0.18 & 0,9948 & 3,2561 & 7,0447 \\
\hline 0.20 & 0,9956 & 3,3178 & 6,9899 \\
\hline \multicolumn{4}{|c|}{$\mathrm{YP}=0.41$} \\
\hline 0.02 & 0,9764 & 1,2615 & 1,4539 \\
\hline 0.04 & 0,9761 & 1,7750 & 2,0384 \\
\hline 0.06 & 0,9774 & 2,1442 & 2,6014 \\
\hline 0.08 & 0,9842 & 1,9617 & 1,9207 \\
\hline 0.10 & 0,9819 & 2,6704 & 3,9885 \\
\hline 0.12 & 0,9865 & 2,5162 & 3,2039 \\
\hline 0.14 & 0,9897 & 2,7532 & 4,2591 \\
\hline 0.16 & 0,9900 & 3,1357 & 5,9392 \\
\hline 0.18 & 0,9949 & 2,9434 & 5,0484 \\
\hline 0.20 & 0,9945 & 3,4697 & 8,2371 \\
\hline \multicolumn{4}{|c|}{$\mathrm{YP}=0.36538$} \\
\hline 0.02 & 0,9801 & 1,2137 & 1,3706 \\
\hline 0.04 & 0,9811 & 1,6596 & 1,8576 \\
\hline 0.06 & 0,9849 & 1,8287 & 2,0027 \\
\hline 0.08 & 0,9865 & 2,0459 & 2,2998 \\
\hline 0.10 & 0,9902 & 2,2034 & 2,7194 \\
\hline 0.12 & 0,9901 & 2,4253 & 3,0788 \\
\hline 0.14 & 0,9912 & 3,4931 & 11,1655 \\
\hline 0.16 & 0,9895 & 2,8963 & 3,9533 \\
\hline 0.18 & 0,9948 & 2,8415 & 4,2569 \\
\hline 0.20 & 0,9973 & 3,1100 & 5,7366 \\
\hline \multicolumn{4}{|c|}{$\mathrm{YP}=0.32143$} \\
\hline 0.02 & 0,9879 & 1,1419 & 1,4300 \\
\hline 0.04 & 0,9877 & 1,4989 & 1,6132 \\
\hline 0.06 & 0,9888 & 1,8653 & 2,3048 \\
\hline 0.08 & 0,9893 & 2,1974 & 2,9570 \\
\hline 0.10 & 0,9920 & 2,2700 & 3,0015 \\
\hline 0.12 & 0,9931 & 2,4636 & 3,4454 \\
\hline 0.14 & 0,9990 & 2,4068 & 3,7823 \\
\hline 0.16 & 0,9975 & 2,7523 & 4,7674 \\
\hline 0.18 & 0,9978 & 2,9100 & 5,1833 \\
\hline 0.20 & 1,0012 & 2,9329 & 5,7425 \\
\hline
\end{tabular}




\begin{tabular}{|c|c|c|c|}
\hline \multicolumn{4}{|c|}{$\mathrm{YP}=0.25$} \\
\hline 0.02 & 0,9905 & 1,0494 & 1,3366 \\
\hline 0.04 & 0,9857 & 1,4598 & 1,3992 \\
\hline 0.06 & 0,9884 & 1,8147 & 2,0989 \\
\hline 0.08 & 0,9908 & 2,0471 & 2,6121 \\
\hline 0.10 & 0,9921 & 2,2934 & 3,1905 \\
\hline 0.12 & 0,9933 & 2,5413 & 3,9981 \\
\hline 0.14 & 0,9957 & 2,6499 & 4,3914 \\
\hline 0.16 & 0,9977 & 2,7733 & 4,8926 \\
\hline 0.18 & 0,9992 & 2,9282 & 5,7158 \\
\hline 0.20 & 0,9993 & 3,1976 & 7,1059 \\
\hline \multicolumn{4}{|c|}{$\mathrm{YP}=0.20588$} \\
\hline 0.02 & 0,9858 & 0,9743 & 1,1775 \\
\hline 0.04 & 0,9834 & 1,4495 & 1,7520 \\
\hline 0.06 & 0,9814 & 1,8316 & 2,1952 \\
\hline 0.08 & 0,9880 & 2,0863 & 3,1540 \\
\hline 0.10 & 0,9900 & 2,1030 & 2,7053 \\
\hline 0.12 & 0,9878 & 2,4669 & 3,5406 \\
\hline 0.14 & 0,9906 & 2,7497 & 4,9152 \\
\hline 0.16 & 0,9972 & 2,6860 & 4,6848 \\
\hline 0.18 & 0,9996 & 2,8356 & 5,4213 \\
\hline 0.20 & 0,9985 & 3,2295 & 7,5611 \\
\hline \multicolumn{4}{|c|}{$\mathrm{YP}=0.125$} \\
\hline 0.02 & 0,9437 & 1,3460 & 1,9438 \\
\hline 0.04 & 0,9429 & 1,8294 & 2,5717 \\
\hline 0.06 & 0,9456 & 2,1444 & 2,7988 \\
\hline 0.08 & 0,9515 & 2,3621 & 3,4842 \\
\hline 0.10 & 0,9561 & 2,6217 & 4,0773 \\
\hline 0.12 & 1,0131 & 2,0387 & 3,4982 \\
\hline 0.14 & 0,9634 & 3,1603 & 6,2314 \\
\hline 0.16 & 0,9873 & 3,0772 & 7,0350 \\
\hline 0.18 & 0,9754 & 3,5081 & 8,1311 \\
\hline 0.20 & 0,9803 & 3,4800 & 8,0012 \\
\hline \multicolumn{4}{|c|}{$\mathrm{YP}=0.10938$} \\
\hline 0.02 & 0,9999 & 0,8582 & 1,1678 \\
\hline 0.04 & 0,9950 & 1,3840 & 1,9839 \\
\hline 0.06 & 0,9938 & 1,5590 & 1,8212 \\
\hline 0.08 & 0,9960 & 1,8727 & 2,6217 \\
\hline 0.10 & 0,9974 & 2,0096 & 2,7001 \\
\hline 0.12 & 0,9981 & 2,2556 & 3,3983 \\
\hline 0.14 & 1,0001 & 2,4253 & 4,0507 \\
\hline 0.16 & 0,9999 & 2,8248 & 5,9333 \\
\hline 0.18 & 1,0017 & 3,6375 & 15,6952 \\
\hline 0.20 & 1,0028 & 2,9581 & 6,2047 \\
\hline \multicolumn{4}{|c|}{$\mathrm{YP}=0.05$} \\
\hline 0.02 & 1,0141 & 0,7503 & 1,1142 \\
\hline 0.04 & 0,9963 & 1,2630 & 1,7780 \\
\hline 0.06 & 1,0006 & 1,4073 & 1,8053 \\
\hline 0.08 & 1,0003 & 1,7002 & 2,3908 \\
\hline 0.10 & 0,9910 & 2,1383 & 3,2723 \\
\hline 0.12 & 1,0175 & 1,9432 & 3,2799 \\
\hline 0.14 & 1,0024 & 2,4523 & 4,5571 \\
\hline 0.16 & 1,0058 & 2,5032 & 4,4276 \\
\hline 0.18 & 1,0039 & 2,9181 & 6,4823 \\
\hline 0.20 & 1,0159 & 2,4694 & 4,0736 \\
\hline
\end{tabular}




\section{CONCLUSION}

The first application of the Variational Monte Carlo methods in quantum mechanical systems was looked at by McMillan [23] investigating bosonic systems. VMC methods were extended to study simple Fermi systems [22,24] and were developed for finite nucleon systems, and these methods have successfully been applied to few nucleon systems $[25,26]$.

In order to obtain properties of many body systems such as nuclear matter, a non-ideal gas etc. the many body problem can be reduced a two-body problem, a three body-problem, etc. Such a decomposition is not applicable to a N-body system since each particle in a $\mathrm{N}$ body system is in constant interaction with large number of its neighbours. Therefore, one have to use the methods that are more suitable for dense systems like nuclear matter. One of these methods is Variational Monte Carlo method.

In this study, as an application of the Variational Monte Carlo method, we have calculated radial distribution functions of nuclear matter. In the calculations, we have used the Urbana $\mathrm{V}_{14}$ potential for the nucleon-nucleon interaction and included the three-body interactions. We have also presented a simple expression for nuclear matter RDF. This expression involves 3 adjustable parameters. One important point is that the new RDF expression presented in this study may use to obtain the thermodynamics properties of nuclear matter in future studies.

\section{REFERENCES}

1. D.A. McQuarrie, Statistical Mechanics, Harper Collins Publishers, New York, 1976.

2. B.J. Alder and C.E. Hecht, Studies in Molecular Dynamics. VII. Hard-Sphere Distribution Functions and an Augmented van der Waals Theory, J. Chem. Phys., 50, p.2032, 1969.

3. J.A. Barker, R.O. Watts and D. Henderson, Monte Carlo values for the radial distribution function of a system of fluid hard spheres, Mol. Phys., 21, p.187, 1971.

4. L. Verlet, Computer "Experiments" on Classical Fluids. II. Equilibrium Correlation Functions, Phys. Rev., 165, p.201, 1968.

5. J.P. Hansen and J.J. Weis, Radial distribution functions for inverse-12 ('soft sphere') fluids, Mol. Phys., 23, p.853, 1972.

6. J.A. Barker, R.A. Fisher and R.O. Watts, Liquid argon: Monte carlo and molecular dynamics calculations, Mol. Phys., 21, p.657, 1971.

7. B.J. Alder and T. Wainwright, In transport processes in statistical mechanics, edited by I. Prigogine, Academic, New York, 1958.

8. A. Morsali, E. K. Goharshadi, G. Ali Mansoori and Mohsen Abbaspour, An accurate expression for radial distribution function of the Lennard-Jones fluid, Chem. Phys. 310, p11-15, 2005.

9. M. Bamdad, S. Alavi, B. Najafi and E. Keshavarzi, A new expression for radial distribution function and infinite shear modulus of Lennard-Jones fluids, Chem. Phys. 325, p554-562, 2006.

10. N. von Solms, K.Y. Koo and Y.C. Chiew, Mixing rules for binary Lennard-Jones chains: theory and Monte Carlo simulation, Fluid Phase Equilibria, 180, p71-85, 2001.

11. B.E. Warren, X-Ray Determination of the Structure of Liquids and Glass, J. Appl. Phys., 8, p.645, 1937. 
12. N.S. Gingrich, The Diffraction of X-Rays by Liquid Elements, Rev. Mod. Phys., 15, p.90, 1943.

13. R.E. Kruh, Diffraction Studies of the Structure of Liquids., Chem. Rev., 62, p.319, 1962.

14. J. Largo and J.R. Solana, A simplified perturbation theory for equilibrium properties of triangular-well fluids, Fluid Phase Equilibria, 167, p.21, 2000.

15. Y. Tang, C. Benjamin and Y. Lu, On the mean spherical approximation for the Lennard-Jones fluid, Fluid Phase Equilibria, 171, p.27, 2000.

16. C. Xi Li, Z. Hao Wang, Y. Gui Li and J. Fang Lu, Two body integrals for hard sphere fluid base on Tang-Lu RDF expression, Fluid Phase Equilibria, 201, p.37, 2002.

17. I.E. Lagaris and V.R. Pandharipande, Phenomenological two-nucleon interaction operator, Nucl. Phys. A, 359, 331, 1981.

18. K. Manisa, U. Atav and R. Oğul, VMC calculations of the ground state properties of nuclear matter, Int. J. of Mod. Phys. E, 14, 255, 2005.

19.K.Manisa, Ü. Atav and S. Sariaydın, Equation of state of asymmetric nuclear matter: a VMC study, Cent. Eur. J. Phys., 8(4), 587-595, 2010.

20. N. Metropolis, A.W. Rosenbluth, M.N. Rosenbluth, A.H. Teller and E. Teller, Equation of State Calculations by Fast Computing Machines, J. Chem. Phys. 21, 1087, 1953.

21. R. Jastrow, Many-Body Problem with Strong Forces, Phys. Rev. 98, 1479, 1955.

22. D. Ceperley, G.V. Chester and M.H. Kalos, Monte Carlo simulation of a manyfermion study, Phys. Rev. B16, 3081, 1977.

23. W.L. McMillan, Phys. Rev. A, 138, 442, 1965.

24. M. A. Lee, K. E. Schmidt, M. H. Kalos and G. V. Chester, Green's Function Monte Carlo Method for Liquid ${ }^{3} \mathrm{He}$, Phys. Rev. Lett., 46, 728, 1981.

25. J. Lomnitz-Adler, V. R. Pandharipande and R. A. Smith, Nucl. Phys. A, 361, 399, 1981.

26. J. Carlson, Alpha particle structure, Phys. Rev. C, 38, 1879, 1988. 